\title{
3D Modeling of Stenotic Internal Carotid Artery Treated with Stent: A CFD Analysis of Blood
}

\author{
M.Elshin Joel ${ }^{1}$, M.Anburajan ${ }^{2}$ \\ ${ }^{1}$ Biomedical Engineer \& Designer, A.I Consultancy \& Services Pvt Ltd, Pune, India, elshinjo.88@gmail.com. \\ ${ }^{2}$ Professor and Head, Department of Biomedical Engineering, SRM University, Chennai-603203, \\ Tamil Nadu, India, Email: hod.biomedi@ktr.srmuniv.ac.in
}

\begin{abstract}
There is enormous evidence that the initiation and growth of atherosclerosis is highly influenced by hemodynamic factors. In this study, hemodynamic factors in the following patient specific 3D carotid artery models were studied: i) normal; ii) stenosis (with plaque near to bifurcation of internal carotid artery (ICA)); and iii) surgical model (plaque with surgically fitted Palmaz-Schatz stent). The 3D model of the carotid artery was formed from CT image of a patient through a commercial software MIMICS. The geometrical measurement taken from the 3D model was used to form its CAD model using CATIA software. The CAD model was meshed in ANSYS ICEM CFD module. The inlet- and outlet- boundary conditions were defined and material properties were given in CFXPre module of ANSYS. Then the solver input file was given to CFX solver manager for performing computational fluid dynamics (CFD) on the model. The outputs of the CFD analysis were observed in CFD-Post. The wall shear stress and blood velocity were greater both at near the bifurcation and at bend of the carotid artery model. The abnormal model is characterized by high wall shear stress in the stenosed portion which indicates greater probability of growth of plaque. Disturbed flow patterns and oscillating velocity indicate that the vulnerability of plaque formation is high in such places. The hemodynamic values have come back near to normal values in the carotid model fitted with stent. The observed variations in hemodynamic factors in the carotid artery with plaque surgically fitted with stent were compared to its normal model. This information can be used to predict restenosis in future.
\end{abstract}

Index Terms - Stenosis; Palmaz-Schatz stent; 3D model of carotid artery; Hemodynamic properties of blood; CFD analysis;

\section{Introduction}

'Carotid artery disease' refers to dysfunction of the carotid artery, which supplies oxygenated blood to neck and head. If a plaque partially blocks the blood flow in carotid artery, the diseased condition is called as 'carotid artery stenosis'. On the other hand, if the plaque blocks completely the carotid artery, the patient is liable to have an increased risk of stroke. Stroke is a major cause of death in India, and in all over the world. The survey of World Health Organization (WHO) states that 15 million people suffer from stroke each year worldwide. Of those, 10 million stroke patients die and the remaining 5 million patients are left permanently disabled. The factors governing blood dynamics such as, its pressure and velocity gradients, flow behavior and its flow rate ratio, velocity distribution, and shear stress on the artery wall and on blood cells are largely responsible for the deposit of cells and lipids, which is a leading cause of atherosclerosis. It was reported that, an artery wall shear stress directly cause the formation of atherosclerosis [5-8]. The deposit of plaque occurs commonly at bifurcations and arterial bends of the carotid artery. In addition to standard systemic risk factors, different responses of vascular endothelium due to altered flow patterns highly contribute in the formation of atherosclerosis [12]. The reasons may be: i) disturbed blood flow; ii) secondary blood flow; and iii) blood flow separation regions. In normal laminar flow, the shear stress maintains thrombo-resistance, barrier function and vascular homeostasis. Blood flow disturbed by low or oscillatory shear stress plays an important role in the formation of atherosclerosis [12]. Giuseppe Patti et al [9] found that the impaired blood mediated dilation independently predicts occurrence of in-stent restenosis. Hence carotid artery simulation study will help in better understanding of the functional, diagnostic and therapeutic aspects of the blood flow. Computational fluid dynamics (CFD) have proven to be a reliable tool for studying three dimensional (3D) flow patterns in complex arterial geometries. In this study, hemodynamic parameters were evaluated in stenois model and stenosis model fitted with stent, when compared to normal carotid model using CFD methodology. Streamline analysis and cut plane contour analysis at specific important locations were done to analyze flow patterns and hemodynamic factors in the anatomically realistic carotid models.

\section{Materials and Methods}

\section{Step-1:MIMICS (Materialise, Belgium)}

A standard computed tomography (CT) image of carotid artery of a normal Indian male aged 37 years was obtained. It had $0.5 \mathrm{~mm}$ resolution, and was in digital imaging and communications in medicine (DICOM) format. When the image was loaded in the Materialise's Interactive Medical Image Control System (MIMICS) software through its import image option, it generated axial, coronal and sagittal portions of the image, automatically. The orientation of the image was changed manually. Based on carotid artery, the region of interest (ROI), thresholding operation was done. The ROI was selected based on Hounsfield unit (HU). It represents the density of the tissue imaged. There are some predefined threshold set values available in the software. They are bone, enamel, soft tissue, compact bone, spongy bone, muscle tissue, fat tissue, and the skin tissue. For selecting carotid artery, bone threshold set value was selected. The dynamic region growing tool allowed segmenting an object based on the connectivity of gray values in a certain gray value range. A new mask was used to select the specific ROI. Further, multiple layer option 
was used to select other slices of images that have connectivity with the selected region and thus the right side carotid artery region was segmented. After selecting specific region by dynamic region growing operation, calculate $3 \mathrm{D}$ icon was used to form 3D model. The required mask to be processed was selected. All manual editing functions were performed on the active mask through edit mask option. The 3D mask editing tool was used to visualize and edit the mask in 3D. After creating 3D model of the carotid artery, various geometric measurements were made.
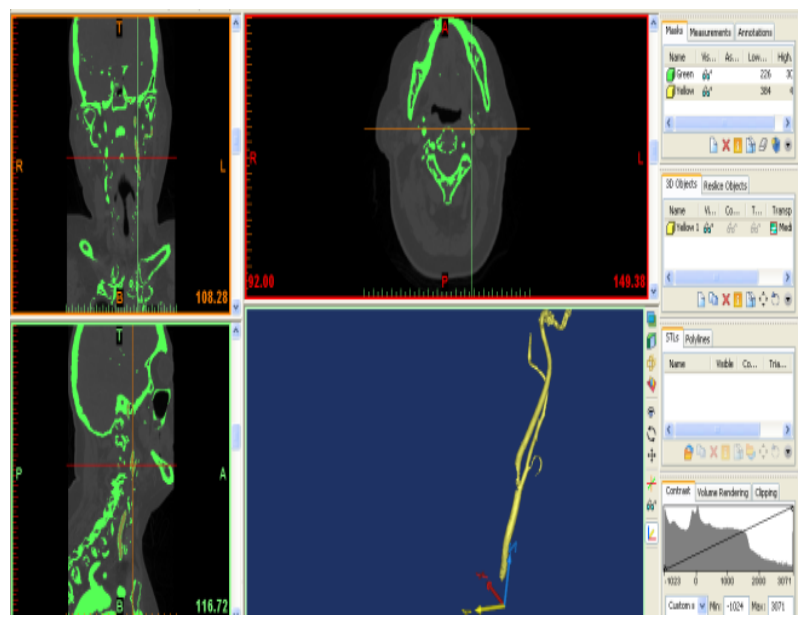

Figure.1. Obtained geometrical model of carotid artery using MIMICS

\section{Step-2: CATIA V5(Dassault systems, France)}

The obtained 3D model was imported to computer aided design (CAD) modelling software CATIA V5. The geometric measurements of different regions of carotid artery were taken from the 3D model created in MIMICS. Now reference points were formed on the geometric model of carotid artery. Planes were created on the reference points and the sketches of different dimensions were drawn on the planes. Splines were used to connect the reference points. Then CAD model of normal carotid artery was formed through reference points along the spline using loft option. The diameter of the artery was varied in each sketch on different points, which was useful in obtaining an anatomically realistic carotid model with varying diameter. The following 3D CAD models of carotid artery were obtained for the study: i).Normal model: carotid artery without any plaque; ii).Stenosis (abnormal) model: carotid artery with a plaque, located near to bifurcation of internal carotid artery (ICA); and iii) Surgical model: carotid artery with plaque at ICA surgically treated with PalmazSchatz stent. After creating CAD models, the files were saved in 'step' format for further analysis using ANSYS software.

\section{Step-3: ANSYS 12.0 (ANSYS, United States of America)}

The CAD model file in 'step' format was imported in ANSYS ICEM CFD module. An inlet and outlet blood flow directions through carotid artery as well as its wall were well defined. The assigned maximum mesh element size for both inlet and outlet of the artery was given as $0.25 \mathrm{~mm}$, whereas, for its wall was given as $0.5 \mathrm{~mm}$. Tetragonal volume meshing was done for all parts created, and the file was saved in ANSYS CFX5 format. Then, the file was imported in ANSYS CFX-Pre. Here, the inlet and outlet of the artery were created and defined. The blood was modelled with density of $1050 \mathrm{Kgm}^{-3}$, viscosity of $0.003 \mathrm{Pas}^{-1}$, and its specific heat capacity of 3780 $\mathrm{Jkg}^{-1} \mathrm{~K}$. After giving the properties, the boundary conditions were defined. The inlet boundary condition was inlet blood velocity, which was given as $0.34 \mathrm{~ms}^{-1}$. The outlet boundary condition was static pressure, which was assigned as '0' Pascal. No slip condition was applied, whereas, smooth wall condition was used to define artery wall roughness. The solver input file was saved in 'def' format.

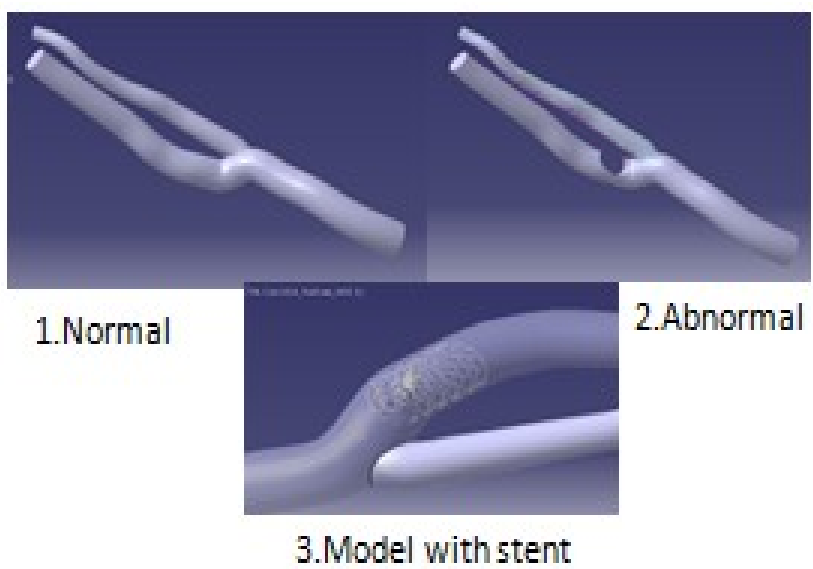

Figure 2. Designed CAD models of CA using CATIA software: i) normal model, without plaque; ii). stenosis model-plaque at bifurcation of internal carotid artery (ICA); and iii) Surgical model-carotid artery with plaque at ICA surgically treated with Palmaz-Schatz stent.

Then the CFX-Solver Manager was opened and the solver input file was selected. The solver manager executed mass and momentum equations on the model with the applied boundary conditions and properties of materials. Then the results was seen in the CFD-Post. The velocity and pressure streamline was seen by selecting them accordingly. The wall shear stress was seen by selecting the wall and changing the variable to wall shear. The evaluated blood pressure, velocity and wall shear stress values were then exported as excel sheets.



Fig.3. Definition of an inlet and outlet boundary conditions in ANSYS CFX-Pre 


\section{Results and Discussion}

The output of the CFD analysis was shown in CFD-Post. The hemodynamic parameters like velocity, pressure and wall shear stress were considered in this study.

\section{A. Approach-I: Streamline Analysis \\ i. Blood velocity}

In normal model, blood velocity was found to be higher at proximal part of both ICA and external carotid artery (ECA). Further, blood velocity was higher in bend and bifurcation regions of carotid artery. In the stenosis model, high blood velocity jet was generated from the stenosis region. The poststenotic flow was highly disordered. It is known that, blood turbulent flow depends on its flow condition and severity of stenosis. In surgical model, blood velocity pattern was seemed to be restored to its normal pattern with the help of stent fitted at stenosis region.

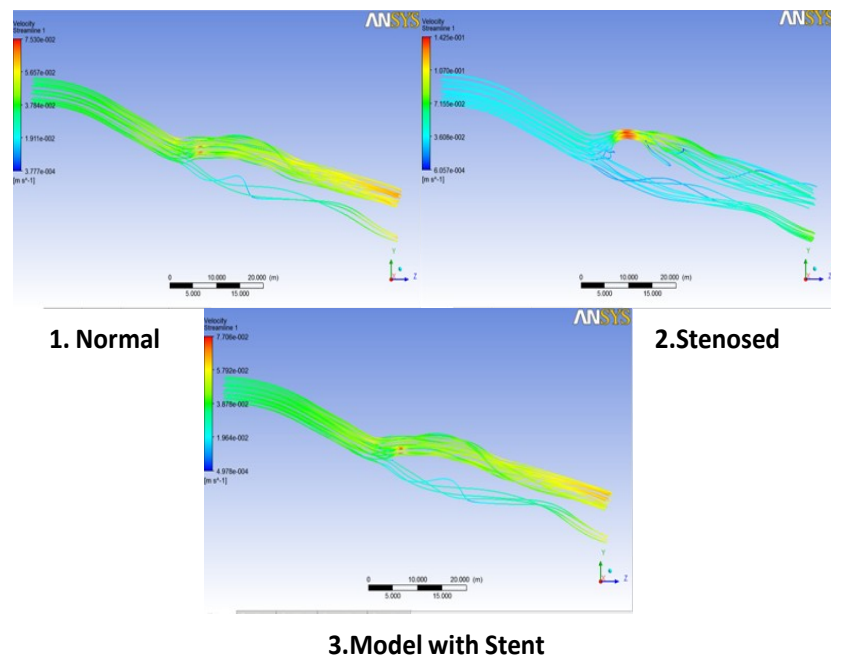

Figure .4.Streamlines analysis of blood velocity in CAD models of carotid artery

\section{ii. Blood pressure}

In normal model, blood pressure was higher at distal part of both ICA and ECA. In stenosis model, blood was accumulated before the plaque region. Hence an increase of blood pressure was observed before the plaque region in the internal carotid artery. Also, blood flow through the distal external carotid artery was greater due to the stenosed internal carotid artery. In surgical model, blood pressure pattern was maintained in the normal range by the fitted stent at stenosis region.

\section{iii. Wall shear stress}

In normal model, wall shear stress was found to be higher in the proximal part of both ICA, and ECA. Further, it was found to be higher in bend and bifurcation regions of carotid artery. On the other hand, in stenosis model, wall shear stress value was found to be highest at plaque region due to occlusion. In surgical model, the wall shear stress regained its normal value, comparing to normal model.



Figure.5. Streamline analysis of blood pressure in CAD models of carotid artery

\section{B. Approach-II: Cut Plane Contour Analysis}

In order to enhance our understanding of blood flow through carotid artery, cut plane contour analysis of both blood velocity and pressure were carried out at three different locations in all three models. In the carotid artery model, a cut plane was taken at the following locations: i).pre-stenotic region at the bifurcation of common carotid artery; ii).stenosis region at distal internal carotid artery, and the corresponding parallel region in external carotid artery; and iii).post-stenotic region in the proximal internal and external carotid artery.



Figure 6. Cut planes at three different locations of the carotid artery model

\section{i. Blood velocity}

In normal model, measured mean blood velocity value had increased progressively along the cut planes, taken at three different pre-defined regions. The measured mean values of blood velocity $(\mathrm{m} / \mathrm{sec})$ at bifurcation, distal- and proximalparts of ICA and ECA were $0.27 \pm 0.12,0.30 \pm 0.11$, and $0.34 \pm 0.11$ respectively. It showed that, the measured standard deviation value of blood velocity $(\mathrm{m} / \mathrm{sec})$ through all the three regions was low. It indicated that there was a uniform blood flow. In stenosis model, blood velocity was higher in the distal part of ICA and ECA (increased from 0.26 at bifurcation to $0.65 \mathrm{~m} / \mathrm{sec}$ at distal part of ICA and ECA). It was found that the pre-stenotic and post-stenotic blood velocity contours were 
disordered, which indicated the presence of secondary flows due to stenosis condition. Also, pre-stenotic blood velocity at the bifurcation slice plane was lesser than that value observed in normal model. Also, an average blood velocity has increased to very high value in the distal stenotic slice plane, followed by decrease in the value in the proximal post-stenotic slice plane. The calculated standard deviation of the blood velocity value in the distal stenotic slice plane was high, which indicated oscillatory blood flow in the stenosis region. In surgical model, measured mean values of blood velocity $(\mathrm{m} / \mathrm{sec})$ at bifurcation, distal- and proximal- parts of ICA and ECA were $0.32 \pm 0.12,0.35 \pm 0.09$, and $0.33 \pm 0.10$ respectively. The observed velocity contour pattern was found to be similar, when compared to its normal model. The calculated mean blood velocity was higher in the distal stenotic slice plane and was slightly lesser in the proximal slice plane.

\section{ii. Blood pressure}

In normal model, measured mean values of blood pressure (Pa) at bifurcation, distal- and proximal- parts of ICA and ECA were $0.020 \pm 0.000,0.017 \pm 0.003$, and $0.016 \pm 0.003$ respectively. It showed that measured mean blood pressure value decreased along the plane. The standard deviation of blood pressure value was less in the bifurcation slice plane. In stenosis model, the standard deviation of blood pressure value was relatively high in proximal- and distal- slice planes. All the cut planes showed significant variations in blood pressure compared to its normal model. It was found that, the blood pressure decreased largely in all the cut planes. The calculated standard deviation value of blood pressure in all the cut planes was high, that indicated turbulent blood flow due to stenosis condition. From the cut plane pressure contour in the distal ICA and ECA, it was seen that the pressure has decreased largely in the stenosed portion of an abnormal carotid model. Also, it was found that the pressure accumulation was higher in its pre-stenotic region (bifurcation). In surgical model, the calculated mean blood pressure value had come back near to the values observed in its normal model. The standard deviation was higher in the bifurcation slice plane compared to the normal model which indicated oscillating pressure due to the placement of stent.

\section{Conclusion}

The hemodynamic parameters like velocity, pressure and wall shear stress had been analysed and compared between the anatomically realistic normal carotid model, stenosis model and stenosis carotid artery surgically treated with stent model. Since the flow pattern is affected by the geometry of the artery, the anatomically realistic artery can be used for accurate prediction of hemodynamic factors. The areas with low wall shear stress values are highly prone to atherosclerosis formation. Areas having very high wall shear stress can also affect the carotid artery by causing endothelial damage which leads to plaque formation. In normal model, wall shear stress was found to be higher in the proximal part of both ICA, and ECA. Further, it was found to be higher in bend and bifurcation regions of carotid artery. On the other hand, in stenosis model, wall shear stress value was found to be highest in plaque region due to occlusion. High oscillation of velocity patterns indicates higher possibility of stenosis formation at such places. The post-stenotic flow was highly oscillating producing secondary flows in those regions. The velocity and wall shear stress was slightly higher in the stenosis model fitted with stent than its normal model indicating the possibility of restenosis in future. Given the set of patterns of flow and values of oscillating blood velocity, pressure and wall shear stress for an anatomical geometry to be healthy, the flow simulation study can help in attaining that healthy state by optimal deployment of stent in the artery. The process of prediction of atherosclerosis can be automated with the numerous set of patterns and values defined for specific condition to be achieved.

\section{References}

[1] E.Aribas, S. Piskin, and M.S. Celebi. 3D blood flow simulation in human arterial tree bifurcations. In Proc. of the IEEE 14th National Biomedical Engineering Meeting (BIY-OMUT, 2009), Izmir, Turkey, 2009, DOI: 10.1109/BIYOMUT.2009.5130389, 2009, pp.1-4

[2] Xu Bai-Nan,Wang Fu-Yu, Liu Lei, Zhang Xiao-Jun, and Ju Hai-Yue, 'Hemodynamics model of fluid-solid interaction in internal carotid artery aneurysms', J.Neurosurg Rev., 2011;34(1): pp.39-47, DOI: $10.1007 / \mathrm{s} 10143-010-0282-5$.

[3] Senol Piskin, Erke Aribas and M. Serdar Celebi. A 3D human carotid artery simulation using realistic geometry and experimental input data. 5th European conference on computational fluid dynamics, ECCOMAS CFD 2010.

[4] D.Martin, A.Zaman, J.Hacker, D.Mendelow and D.Birchall. Analysis of haemodynamic factors involved in carotid atherosclerosis using computational fluid dynamics. The British Journal of Radiology, 82 (2009), S33-S3

[5] C.G.Caro, J.M.Fitz-Gerald, R.C.Schroter. Arterial wall shear stress and distribution of early atheroma in man. Nature 1969; 223: pp.1159-1161

[6] M.H.Friedman, G.M.Hutchins, C.B.Bargeron, O.J.Deters, F.F.Mark. 'Correlation between intimal thickness and fluid shear in human arteries', Atherosclerosis 1981; 39: pp.425 -436

[7] D.N.Ku, D.P.Giddens, C.K.Zarins, S.Glagov, 'Pulsatile flow and atherosclerosis in the human carotid bifurcation: positive correlation between plaque location and low and oscillating shear stress', Arteriosclerosis 1985;5: pp.293-302

[8] Zarins CK, Giddens DP, Bharadvaj BK, Sottiurai VS, Mabon RF, Glagov S. Carotid bifurcation atherosclerosis: quantitative correlation of plaque localization with flow velocity profiles and wall shear stress. Circ Res1983;53:502 -514

[9] G.Patti, V.Pasceri, R.Melfi, C.Goffredo, M.Chello, A.D’Ambrosio, R.Montesanti, G.Di Sciascio, 'Impaired Flow-Mediated Dilation and Risk of Restenosis in Patients Undergoing Coronary Stent Implantation. Circulation, 2005; 111: pp.70-75, DOI:10.1161/ 01. CIR. 0000151308. 06673.D2

[10] Gnasso A, Irace C, Carallo C, De Franceschi MS, Mottic,Mattioli PL, et al. In vivo association between low wall shear stress and plaque in subjects with asymmetric carotid atherosclerosis. Stroke 1997; 28: pp.993-8.

[11] M.Motomiya, T.Karino, 'Flow patterns in the human carotid artery bifurcation', Stroke 1984;15: pp.50-6.

[12] Kristopher S Cunningham and Avrum I Gotlieb. The role of shear stress in atherosclerosis. Laboratory Investigation, 2005; 85, pp.9-23. 